\title{
Myocardial changes on 3T cardiovascular magnetic resonance imaging in response to haemodialysis with fluid removal
}

\author{
Alastair J. Rankin ${ }^{1 *} \mathbb{D}$, Kenneth Mangion ${ }^{1}$, Jennifer S. Lees ${ }^{1}$, Elaine Rutherford ${ }^{1}$, Keith A. Gillis ${ }^{2}$, Elbert Edy ${ }^{1}$, \\ Laura Dymock ${ }^{3}$, Thomas A. Treibel ${ }^{4}$, Aleksandra Radjenovic ${ }^{1}$, Rajan K. Patel ${ }^{2}$, Colin Berry' ${ }^{1}$, Giles Roditi ${ }^{1,5}$ and \\ Patrick B. Mark'
}

\begin{abstract}
Background: Mapping of left ventricular (LV) native T1 is a promising non-invasive, non-contrast imaging biomarker. Native myocardial T1 times are prolonged in patients requiring dialysis, but there are concerns that the dialysis process and fluctuating fluid status may confound results in this population. We aimed to assess the changes in cardiac parameters on $3 \mathrm{~T}$ cardiovascular magnetic resonance (CMR) before and after haemodialysis, with a specific focus on native T1 mapping.

Methods: This is a single centre, prospective observational study in which maintenance haemodialysis patients underwent CMR before and after dialysis (both scans within $24 \mathrm{~h}$ ). Weight measurement, bio-impedance body composition monitoring, haemodialysis details and fluid intake were recorded. CMR protocol included cine imaging and mapping native $\mathrm{T} 1$ and $\mathrm{T} 2$.

Results: Twenty-six participants (16 male, $65 \pm 9$ years) were included in the analysis. The median net ultrafiltration volume on dialysis was $2.3 \mathrm{~L}(\mathrm{IQR} 1.8,2.5)$, resulting in a median weight reduction at post-dialysis scan of $1.35 \mathrm{~kg}$ (IQR $1.0,1.9$ ), with a median reduction in over-hydration (as measured by bioimpedance) of $0.75 \mathrm{~L}$ (IQR 0.5, 1.4). Significant reductions were observed in LV end-diastolic volume $(-25 \mathrm{ml}, p=0.002)$, LV stroke volume $(-13 \mathrm{ml}, p=0.007)$, global T1 (21 ms, $p=0.02)$, global T2 $(-1.2 \mathrm{~ms}, p=0.02)$ following dialysis. There was no change in LV mass $(p=0.35)$, LV ejection fraction $(p=0.13)$ or global longitudinal strain $(p=0.22)$. On linear regression there was no association between baseline over-hydration (as defined by bioimpedance) and global native T1 or global T2, nor was there an association between the change in over-hydration and the change in these parameters.

Conclusions: Acute changes in cardiac volumes and myocardial native T1 are detectable on 3T CMR following haemodialysis with fluid removal. The reduction in global T1 suggests that the abnormal native T1 observed in patients on haemodialysis is not entirely due to myocardial fibrosis.
\end{abstract}

Keywords: End-stage kidney disease, Haemodialysis, Cardiovascular, Magnetic resonance imaging, Left ventricular hypertrophy

*Correspondence: Alastair.Rankin@glasgow.ac.uk

${ }^{1}$ Institute of Cardiovascular and Medical Sciences, University of Glasgow, 126 University Place, Glasgow G12 8TA, UK

Full list of author information is available at the end of the article

\section{Introduction}

Patients with chronic kidney disease (CKD) are at a greatly increased risk of cardiovascular disease (CVD) [1]. This risk increases with severity of CKD [2], such that patients with CKD stage 5 are 3-4 times more likely to original author(s) and the source, provide a link to the Creative Commons licence, and indicate if changes were made. The images or other third party material in this article are included in the article's Creative Commons licence, unless indicated otherwise in a credit line to the material. If material is not included in the article's Creative Commons licence and your intended use is not permitted by statutory regulation or exceeds the permitted use, you will need to obtain permission directly from the copyright holder. To view a copy of this licence, visit http://creativecommons.org/licenses/by/4.0/. The Creative Commons Public Domain Dedication waiver (http://creativeco mmons.org/publicdomain/zero/1.0/) applies to the data made available in this article, unless otherwise stated in a credit line to the data. 
experience a cardiovascular event than age-standardized patients without CKD [3]. In patients with kidney disease receiving dialysis, CVD remains the single most common cause of death, accounting for between 25 and 40\% of all deaths [4-6]. CKD results in a unique cardiovascular phenotype; with relatively fewer deaths due to atherosclerotic processes and more due to sudden cardiac death and heart failure [4-8]. Cardiomyopathy of CKD, often called 'uraemic cardiomyopathy', refers to a specific pattern of myocardial fibrosis, left ventricular (LV) hypertrophy and diastolic dysfunction, which is found in patients with CKD and forms the pathological basis for this unique CVD phenotype [9-11].

Cardiovascular magnetic resonance imaging (CMR) is established as the reference method for imaging uraemic cardiomyopathy [12]. Previous studies using gadoliniumenhanced CMR demonstrated the presence of myocardial fibrosis in patients on dialysis [13], and its association with poor survival [14]. However, the discovery of the association between gadolinium based contrast media in patients with CKD and the development of the very rare disease nephrogenic systemic fibrosis curtailed further research using this technique $[15,16]$. There is a pressing need for an alternative marker of uraemic cardiomyopathy, further intensified by the observation that regression of LV mass in isolation may not be robustly associated with improved CVD outcomes in patients with CKD [17]. Attempts to identify reliable imaging biomarkers in this population are hampered by the potential confounding influence of the dialysis process itself and fluctuating fluid status.

Native T1 mapping is a non-contrast technique that estimates myocardial longitudinal relaxation times (ms) and reflects changes in extra- and intra-cellular compartments. Myocardial T1 is commonly affected by changes in collagen (fibrosis), water (oedema), iron deposition (haemochromatosis, myocardial haemorrhage) and lipids (Anderson-Fabry's disease) [18]. In addition, native T1 mapping has been shown to differentiate dialysis patients from both healthy [19] and co-morbid controls [20], with excellent inter-observer reproducibility [20, 21]. Outside of the CKD population, T1 mapping has been shown to correlate well with myocardial fibrosis in other disease states [12, 22, 23]. However, the major concern with using native T1 mapping in dialysis patients is the potential confounding influence on the T1 signal of changing tissue oedema resulting from the large intradialytic fluid fluctuations that are typical of patients on intermittent haemodialysis [24]. A previous study using 1.5T CMR observed small, but detectable, differences in native $\mathrm{T} 1$ times immediately after haemodialysis [25]. In the present study we assess the myocardial changes on 3T CMR in response to haemodialysis with fluid removal, with a particular interest in native T1 to inform its potential suitability as a surrogate outcome measure in future therapeutic trials. We also explored the potential bias of dialysis timing in relation to the clinical applicability of 3T CMR.

\section{Materials and methods \\ Participants}

Participants were aged $>40$ years and were established on regular, day-time hospital-based haemodialysis for at least 6 months. Participants were eligible for inclusion if they had a history of recurrent fluid overload (defined as requirement for ultrafiltration volumes of at least 1.5 $\mathrm{L}$ mean fluid removal over the preceding 3 dialysis sessions) and without heart failure (defined as no previous clinical diagnosis of heart failure or with preserved LV ejection fraction (LVEF) $(>50 \%)$ on their most recent transthoracic echocardiogram). Participants had to be able to comply with study procedures, self-report an ability to lie flat for $1 \mathrm{~h}$ and provide informed consent. Exclusion criteria included standard contra-indications to CMR and contraindications to iodine based radiological contrast (to facilitate a sub-study comparing CMR with a novel contrast CT technique) $[26,27]$. The study was prospectively registered at clinicaltrials.gov (NCT03704701). Favourable ethical opinion was granted by the West of Scotland Research Ethics Committee 1 (Ref: 18/ WS/0138, 13th August 2018). All study procedures were carried out in accordance with local guidelines and regulations and with respect to the Declaration of Helsinki.

\section{Study protocol}

This single centre observational study consisted of 2 visits (Fig. 1). Visit 1 occurred before a participant's routine dialysis session. Where possible, this occurred at the end of a participant's 'long', or two-day, gap, i.e., on a Monday for participants on a Monday, Wednesday, Friday dialysis schedule. Participants on a morning dialysis schedule attended visit 1 the afternoon before dialysis. Between visits 1 and 2 participants were asked to consume food and drink as they normally would but to document what they had taken. Participants would then attend their routine haemodialysis session which was performed as per usual clinical practice. Details of the dialysis session were recorded including duration, ultrafiltration volume, settings and medications administered. Visit 2 occurred after dialysis. Participants on an afternoon dialysis schedule attended visit 2 the following morning. At each visit, weight measurement, bioimpedance body composition monitoring (using a Fresenius Body Composition Monitor, Fresenius Medical Care, Hong Kong as per manufacturer's instructions), blood tests and CMR were performed. 

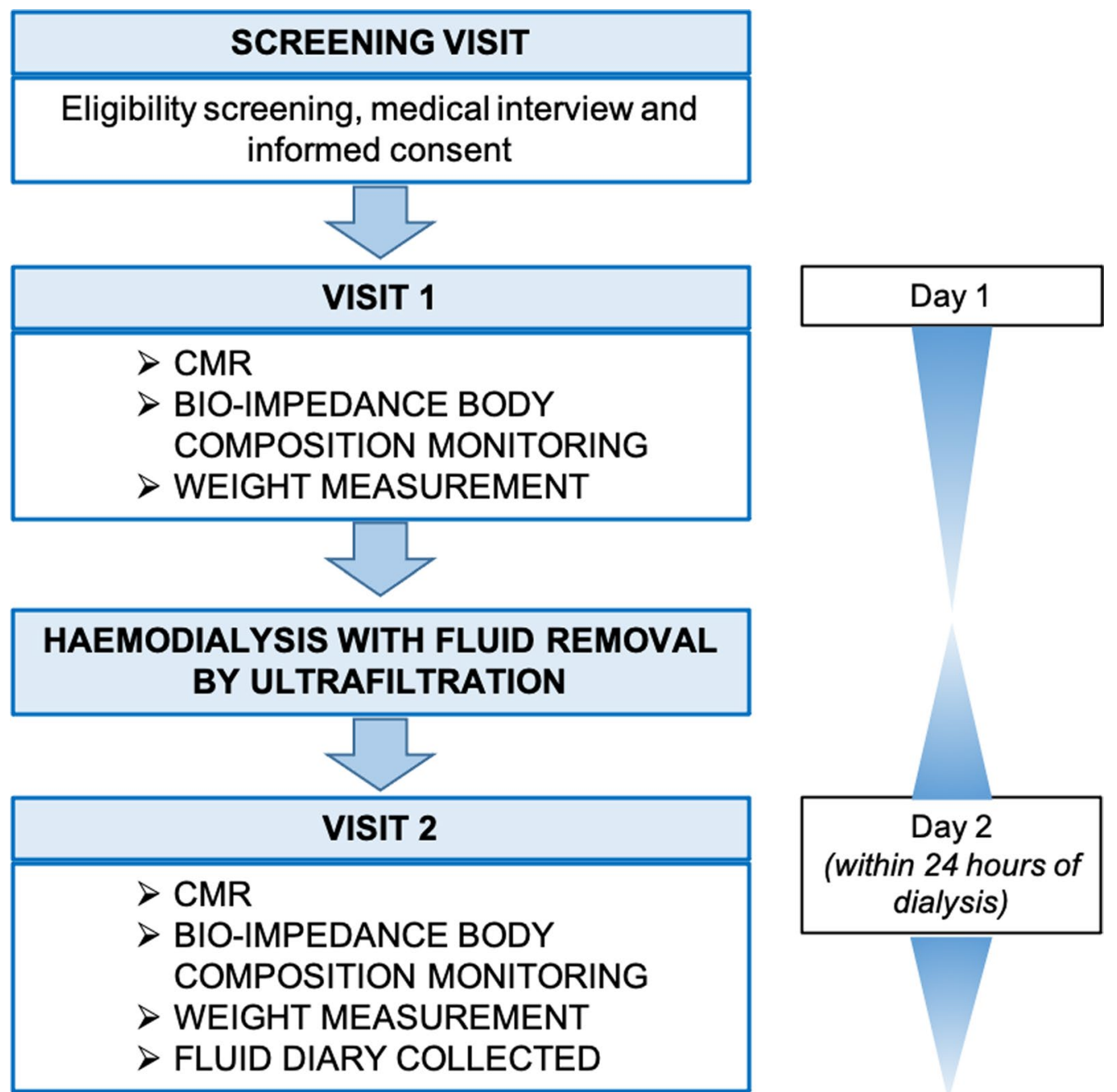

Fig. 1 Flow chart depicting study procedures

\section{CMR image acquisition}

CMR acquisition was performed at the Clinical Research Imaging Facility of the Queen Elizabeth University Hospital in Glasgow using a $3 \mathrm{~T}$ CMR scanner (PRISMA, Siemens Healthineers, Erlangen, Germany) with an 18-channel surface coil placed anteriorly and a 32-channel spine coil placed posteriorly. Following the acquisition of localiser images, balanced steady state free precession sequences were used to acquire LV cine imaging in three long axis planes, followed by a short axis stack from the apex to the atrio-ventricular ring, each with 25 phases. Images were obtained using retrospective electrocardiogram (ECG)-gating at end-expiration. Where participants were unable to breath-hold or had cardiac arrhythmia, compressed sensing (CS cardiac Cine, Siemens Healthineers) was used to allow real-time acquisition. Typical scan parameters were: field of view (FOV) $340 \times 286 \mathrm{~mm}$, slice thickness $7 \mathrm{~mm}$ with $3 \mathrm{~mm}$ gap in short axis stack, repetition time (TR) $-41.4 \mathrm{~ms}$, echo time (TE) $1.51 \mathrm{~ms}$, flip angle $50^{\circ}$, voxel size $1.33 \times 1.33 \times 7 \mathrm{~mm}$.

For native T1 and T2 mapping, basal, mid and apical short axis views were acquired using SiemensMyoMaps sequences. For native T1, non-contrast, motion-corrected, optimized, modified Look-Locker inversion recovery sequences were used with the following typical parameters: FOV $340 \times 272 \mathrm{~mm}$, slice thickness $6.0 \mathrm{~mm}$, voxel size: $1.9 \times 1.9 \times 6.0 \mathrm{~mm}$, TR $272 \mathrm{~ms}$, TE $1.12 \mathrm{~ms}$, flip angle 35 degrees, minimum T1 $100 \mathrm{~ms}$, inversion-time increment $80 \mathrm{~ms}$, bandwidth $1085 \mathrm{Hertz} /$ pixel. For T2 mapping, three T2 weighted measurements were acquired followed by an automated exponential fit for each pixel after respiratory motion correction. The imaging used a T2-prepared single shot 
balanced steady state free precession readout with T2 preparation times $(\mathrm{TE})=0,25$, and $55 \mathrm{~ms}$ with a recovery period of 3 heartbeats between measurements. Typical protocol parameters for T2 mapping were: FOV $360 \times 270 \mathrm{~mm}$, slice thickness $8 \mathrm{~mm}$, matrix $192 \times 108$, spatial resolution $1.9 \times 2.5 \mathrm{~mm}$, TR $207.39 \mathrm{~ms}$, TE $1.32 \mathrm{~ms}$, flip angle 12 degrees, bandwidth $1184 \mathrm{~Hz} /$ pixel.

\section{CMR image analysis}

All CMR scans were subject to a clinical report for clinical governance purposes. Research CMR analysis was performed utilizing dedicated CMR software (cvi42, version 5.10, Circle Cardiovascular Imaging, Calgary, Alberta, Canada)). Routinely reported CMR measures of $\mathrm{LV}$ and right ventricular (RV) function were carried out according to current guidelines [28]. Parameters of myocardial mass and volumes were not indexed to body surface area to avoid confounding impact of weight changes falsely adjusting body surface area and is acceptable given the analysis of within-subject comparisons. Ventricular endocardial and epicardial contours were manually drawn at end-diastole (Fig. 2). LV endocardial contours were drawn at end-systole, which was deemed to be the phase with the smallest blood pool cavity. Papillary muscles were excluded from myocardial mass and included in volumes. LV thickness was recorded as the maximum septal thickness measured perpendicular to the cavity on a short-axis mid-chamber view, at the approximate level of the mitral valve leaflet tips. Global LV strain (circumferential, longitudinal, and radial) and global RV strain (longitudinal and radial) were derived using the software's tissue tracking module to determine peak values for each parameter. Atrial volumes were manually drawn on 4-chamber horizontal long axis views at atrial systole and diastole (defined with respect to mitral valve closure) to report maximum and minimum right atrial (RA) volumes and atrial emptying fraction. For left atrial (LA) measurements, the vertical long axis views were additionally contoured to report biplanar derived values. For T1 and T2 measurements, scanner derived maps were used. Epi- and endocardial borders were manually drawn on each basal, mid and apical map. Areas of obvious artefact were excluded from regions of interest (ROI) and care was taken to include only myocardial tissue with a $10 \%$ epi- and endocardial offset applied. Global values were derived by averaging results from all three short axis slices. Septal values were reported as the mean of segments 2, 3, 8, 9 and 14 as per the American Heart Association's 16 segment model [29]. For blood pool T1 and T2, ROIs were drawn within the LV cavity on the mid-LV map, with care taken to avoid artefact and papillary muscles. Additional ROIs were manually drawn on a representative area of skeletal muscle, with the pectoralis major muscle used preferentially. A further ROI was drawn within a homogenous region within the right lobe of liver. The primary observer (AJR) batch analysed all CMRs in a random order and was blinded to participant identity and whether the scan was pre-or post-dialysis. A second independent observer (KM) analysed a random sample of $>20 \%$ of the cohort to assess inter-observer variability. As a post-hoc experiment, a T1MES phantom [30] was scanned on consecutive days at times that replicated the study schedule to assess inter-study $\mathrm{T} 1$ variability.

\section{Statistical analysis}

Continuous data with a normal distribution are presented as mean \pm standard deviation (SD), and median and interquartile range (IQR) for skewed data, with normality defined according to Shapiro-Wilk test. Pre and post dialysis CMR values were compared using paired t-tests and Wilcoxon singed rank tests accordingly. Linear regression and multiple regression were used to compare change in CMR parameters according to baseline variables. Repeated measures MANCOVA was used to account for covariates in the comparison of myocardial native $\mathrm{T} 1$ before and after dialysis. Intra- and interobserver variability was assessed by the intra-class correlation (ICC) coefficient (two-way mixed effect, average measures). Statistical analysis was performed, and figures created, using SPSS (version 27, Statistical Package for the Social Sciences, International Business Machines, Inc., Armonk, New York, USA).

\section{Sample size}

A prospective sample size calculation determined that a total of 9 participants would be required to detect a $2.5 \%$ difference in native $\mathrm{T} 1$ times with $80 \%$ power and alpha 0.05 based on previously published data $[19,21]$. A total of 22 participants would be sufficient to detect a $1.5 \%$ difference. A target of 30 participants was set to allow drop out and to facilitate a pre-specified sub-study (clinicaltrials.gov NCT03704701).

\section{Results}

\section{Participant characteristics}

Twenty-eight participants were recruited between 19th October 2018 and 9th March 2020. Recruitment was stopped early (target $n=30$ ) due to the COVID-19 pandemic. Two participants withdrew consent prior to any study procedures leaving 26 for analysis, of whom $16(61.5 \%)$ were male, $22(84.6 \%)$ were white and age $64.7 \pm 9.4$ years. Median duration of kidney replacement therapy at time of recruitment was $2.0(1.3,4.0)$ years. Multi-morbidity was prevalent in the cohort with a mean 


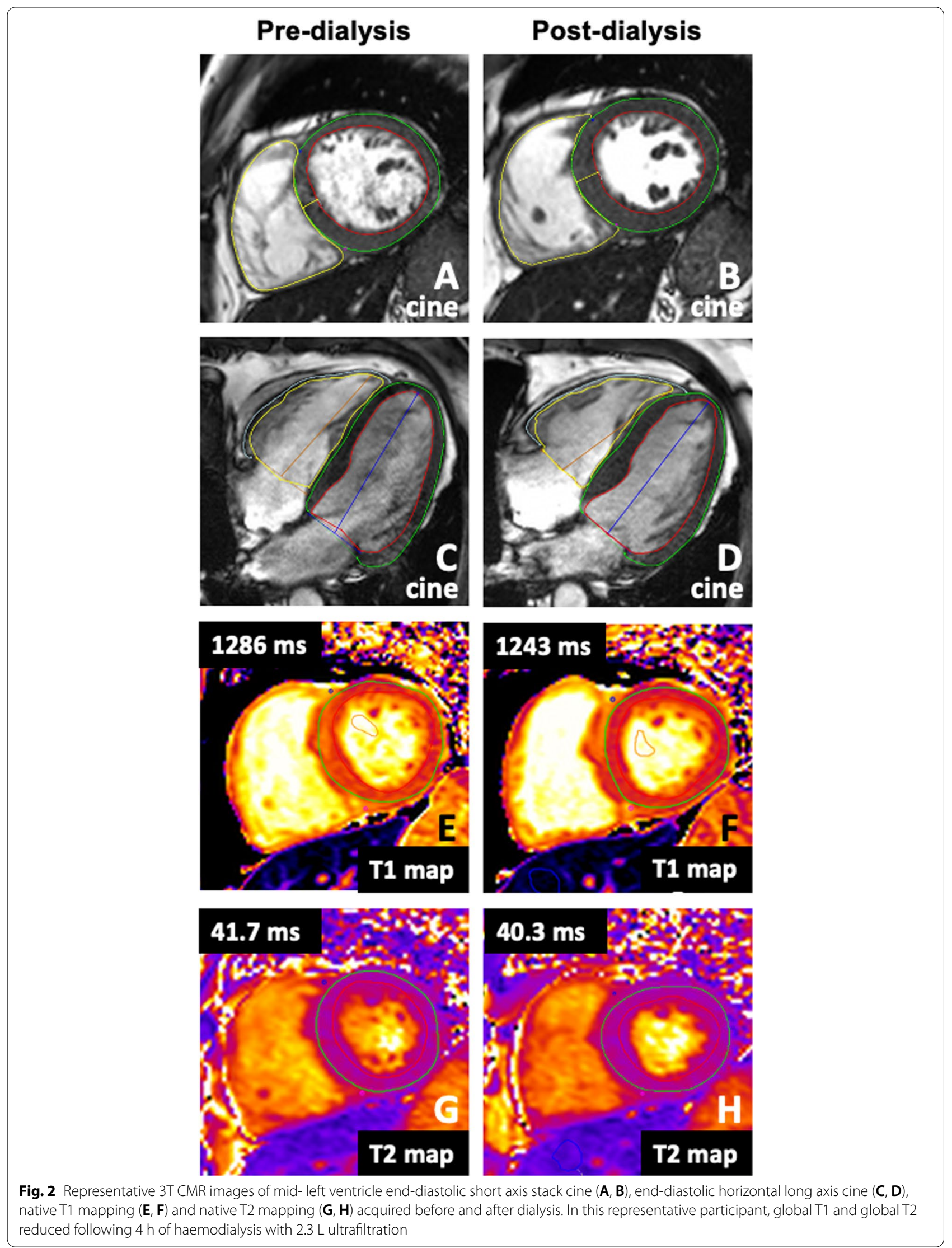


Charlson Comorbidity index of 6 (mean modified Charlson Comorbidity Index of 3) [31, 32]. The median duration of haemodialysis treatment session was $4.0 \mathrm{~h}$ (4.0, $5.0)$ with a mean blood flow of $265( \pm 32) \mathrm{ml} / \mathrm{min}$. Eighteen $(69.2 \%)$ participants followed an afternoon dialysis schedule and underwent dialysis median $2.5(2.0,2.8)$ hours after their first CMR, with repeat CMR at median $15.3(14.8,16.7)$ hours after completion of dialysis. The remaining $8(30.8 \%)$ participants followed a morning dialysis schedule and underwent dialysis at median 16 (14.7, 16.2) hours after their first CMR, with a repeat CMR $1.5(1.2,2.7)$ hours after completing dialysis. For 23 (88.5\%) participants, visit 1 took place after their 'long gap' between dialysis sessions (i.e., pre-dialysis on a Monday for a patient on a Monday, Wednesday, Friday dialysis schedule). Additional baseline characteristics are detailed in Additional file 1: Table S1. In 6 participants, clinically significant incidental findings were detected, including 2 cancers requiring treatment (Additional file 1: Table S2).

\section{Fluid status}

All participants had a history of recurrent fluid overload with mean ultrafiltration volume of $2.2 \mathrm{~L}( \pm 0.4)$ from the preceding 3 dialysis sessions prior to recruitment. At visit 1 (pre-dialysis), 12 participants had demonstrable pitting oedema. 1 participant was unable to undergo bioimpedance monitoring for multifactorial reasons (body habitus, immobility, skin emollient). Of the remaining 25 participants, the median over-hydration was +0.4 $\mathrm{L}(-2.8,+3.5)$, with 10 participants measuring as volume deplete pre-dialysis. The median net ultrafiltration volume on dialysis was $2.3 \mathrm{~L}(1.8,2.5)$ at a mean rate of $6 \mathrm{~mL} / \mathrm{kg} / \mathrm{h}( \pm 1.7)$. Five participants experienced symptomatic intradialytic hypotension requiring adjustment of their dialysis prescription. Between visit 1 (pre-dialysis) and visit 2 (post-dialysis), the median estimated fluid intake was $0.9 \mathrm{~L}(0.6,1.0)$. At visit 2 (post-dialysis), the median reduction in body weight was $1.4 \mathrm{~kg}(1.0$, 1.9), with a median reduction in over-hydration of $0.8 \mathrm{~L}$ $(0.5,1.4)$. No participants gained weight between visit 1 and visit 2, albeit 2 participants' weight did not change. According to bioimpedance monitoring, 3 participants increased their over-hydration between visits (range 0.2$0.4 \mathrm{~L})$.

\section{CMR parameters pre- and post-dialysis}

Table 1 shows the CMR results before and after dialysis. Notable findings include a significant reduction in LV end-diastolic volume, LV stroke volume, RV stroke volume, LA volumes, global circumferential strain, global native T1, septal native T1 and global T2 following dialysis. There was no change in LV mass, LV or RV ejection fraction or global longitudinal strain (Table 1). Figure 3 shows within-subject changes for LV mass, LVEF, LA maximum volume, global T1, septal T1 and global T2. The intra- and interobserver reproducibility for global T1 was excellent with ICC of 0.989 and 0.949 , respectively. Additional intra- and interobserver reproducibility results are included in Additional file 1: Table S3.

\section{Change in native $\mathrm{T} 1$ and $\mathrm{T} 2$ by fluid status}

On linear regression there was no relationship between baseline over-hydration and global native T1, septal T1 or global T2 (Fig. 4). There was also no relationship between the change in global T1, septal T1 or global T2 with ultrafiltration volume $(\mathrm{p}=0.88)$, change in over-hydration $(\mathrm{p}=0.87)$ or change in weight $(\mathrm{p}=0.95)$ (Fig. 4). There was no difference in the mean change in global native T1, septal T1 and global T2 in individuals who did versus did not achieve $>0.5 \mathrm{~L}$ reduction in overhydration (change in global T1: 11.3 (95\% CI - 30.2, 52.8), $\mathrm{p}=0.58$; septal T1: $0.24(95 \% \mathrm{CI}-41.8,42.3)$, $\mathrm{p}=0.99$; global T2: $-0.65(95 \% \mathrm{CI}-2.1,0.81), \mathrm{p}=0.37)$ nor in those with or without $>1.0 \mathrm{~kg}$ weight change (change in global T1: 6.3, $(95 \% \mathrm{CI}-39.4,52.0) \mathrm{p}=0.78$; septal T1: $1.2(95 \% \mathrm{CI}-45.6,48.1), \mathrm{p}=0.95$; global T2: $0.09,(95 \% \mathrm{CI}-1.5,1.7), \mathrm{p}=0.91)$. Blood pool native $\mathrm{T} 1$ correlated with the degree of overhydration measured on bioimpedance at baseline $\left(r^{2}=0.247, p=0.013\right)$ but there was no association between the change in blood pool T1 and the change in overhydration. There was also no correlation between the change in in myocardial native $\mathrm{T} 1$ time and the change in blood pool T1 $(\mathrm{r}=0.13, \mathrm{p}=0.54)$, nor the change in haematocrit $(r=-0.25, p=0.22)$. On repeated measures MANCOVA, when the change in blood pool T1 and the change in haematocrit were added as covariates to the comparison of myocardial native $\mathrm{T} 1$ time, there was no significant interaction between either covariate and the change in myocardial native T1. Both covariates had small, non-significant contributions to the observed effect (change in blood pool T1 (partial eta squared 0.06, $p=0.72$ ); change in haematocrit (partial eta squared $0.11, \mathrm{p}=0.11$ ), resulting in an adjusted $\mathrm{p}$-value of 0.050 for the comparison of myocardial naïve T1 before and after dialysis. Additional determinants of blood T1 are examined in Additional file 1: Table S4.

\section{Change in native $\mathrm{T} 1$ and $\mathrm{T} 2$ parameters by dialysis session}

There was no association between the change in global native $\mathrm{T} 1$, septal $\mathrm{T} 1$ or global $\mathrm{T} 2$ and the time from dialysis until repeat CMR $(\mathrm{p}=0.80,0.55$ and 0.77 respectively, when mean change in values was compared between morning and afternoon dialysis patients). Eighteen participants were on a morning dialysis schedule, whereas 8 were on an afternoon 
Table 1 Cardiovascular Magnetic resonance (CMR) parameters pre- and post-dialysis

\begin{tabular}{|c|c|c|c|}
\hline CMR parameter & Pre-dialysis & Post-dialysis & p-value \\
\hline LV myocardial mass (g) & $103.8(78.8,142.4)$ & $97.5(78.2,136.0)$ & 0.35 \\
\hline LV end diastolic volume (ml) & $185(159,229)$ & $160(152,220)$ & 0.002 \\
\hline LV end systolic volume (ml) & $88(71,113)$ & $84(69,111)$ & 0.81 \\
\hline LV stroke volume (ml) & $103( \pm 29)$ & $90( \pm 30)$ & 0.007 \\
\hline LV ejection fraction (\%) & $53.6(48.6,59.5)$ & $49.8(46.2,54.5)$ & 0.13 \\
\hline LV global longitudinal strain (\%) & $-13.8( \pm 3.3)$ & $-13.1( \pm 3.6)$ & 0.22 \\
\hline LV global circumferential strain (\%) & $-16.3(-19.5,-14.0)$ & $-15.1(-16.9,-13.4)$ & 0.03 \\
\hline LV global radial strain (\%) & $22.2( \pm 6.7)$ & $20.7( \pm 7.1)$ & 0.18 \\
\hline LV thickness (mm) & $10.2(8.4,12.2)$ & $10.6(8.8,12.3)$ & 0.44 \\
\hline RV end diastolic volume (ml) & $161(133,184)$ & $136(128,171)$ & $<0.001$ \\
\hline RV end systolic volume (ml) & $67(56,82)$ & $62(53,75)$ & 0.66 \\
\hline RV stroke volume (ml) & $98( \pm 30)$ & $84( \pm 26)$ & $<0.001$ \\
\hline RV ejection fraction (\%) & $56.9( \pm 10.5)$ & $53.8( \pm 12.6)$ & 0.05 \\
\hline RV global longitudinal strain (\%) & $-22.5( \pm 5.9)$ & $-22.4( \pm 6.8)$ & 0.88 \\
\hline RV global radial strain (\%) & $48.3(37.2,66.7)$ & $49.8(40.0,71.7)$ & 0.77 \\
\hline Minimum LA volume (ml) & $44(28,70)$ & $40(22,70)$ & 0.001 \\
\hline Maximum LA volume (ml) & $96(75,108)$ & $86(57,101)$ & $<0.001$ \\
\hline Minimum RA volume (ml) & $30(20,44)$ & $29(22,41)$ & 0.95 \\
\hline Maximum RA volume (ml) & $60(49,77)$ & $54(45,75)$ & 0.09 \\
\hline Global native T1 (ms) & $1283( \pm 51)$ & $1262( \pm 49)$ & 0.02 \\
\hline Septal native T1 (ms) & $1313( \pm 54)$ & $1293( \pm 47)$ & 0.04 \\
\hline Blood pool native T1 (ms) & $1957( \pm 68)$ & $1935( \pm 73)$ & 0.08 \\
\hline Skeletal muscle native T1 (ms) & $1218( \pm 65)$ & $1210( \pm 73)$ & 0.60 \\
\hline Liver native T1 (ms) & $686( \pm 157)$ & $679( \pm 146)$ & 0.45 \\
\hline Global T2 (ms) & $42.2(40.9,44.8)$ & $41.0(39.9,44.7)$ & 0.02 \\
\hline Blood pool T2 (ms) & $101.1( \pm 20.9)$ & $111.0( \pm 24.5)$ & 0.06 \\
\hline Skeletal muscle T2 (ms) & $32.2( \pm 2.1)$ & $30.8( \pm 3.0)$ & 0.03 \\
\hline Liver T2 (ms) & $21.6(19.9,23.5)$ & $21.3(20.1,22.4)$ & 0.81 \\
\hline
\end{tabular}

Displayed as mean, standard deviation and paired t-test for variables with a normal distribution, and median, interquartile range and Wilcoxon signed rank test for those with a skewed distribution

The scanner-specific reference range for myocardial native global $\mathrm{T1}$ in healthy subjects is mean (range) $1170 \mathrm{~ms}$ (1107-1234) and global T2 is mean $39.5 \mathrm{~ms}$ (34.7-44.3) (unpublished data, correspondence from Dr Kenneth Mangion and Dr Andrew Morrow)

$L V$ left ventricular, $R V$ right ventricular, $L A$ left atrial, $R A$ right atrial

schedule and thus scanned at different times of day. The mean difference in myocardial native $\mathrm{T} 1$ pre/post dialysis was $25 \mathrm{~ms}$ in the morning group $(\mathrm{n}=8)$ and $20 \mathrm{~ms}$ in the afternoon group $(\mathrm{n}=18)$, with no significant difference between the groups $(p=0.80)$. When the T1MES phantom was scanned on consecutive days the measured $\mathrm{T} 1$ was $1216 \pm 8 \mathrm{~ms}$ and $1215 \pm 13 \mathrm{~ms}$, respectively. The same values for T2 were $80.8 \pm 0.9 \mathrm{~ms}$ and $80.2 \pm 1.9 \mathrm{~ms}$. There was no difference in global T1, septal T1 or global T2 in those participants who experienced symptomatic intradialytic hypotension versus those who did not $(\mathrm{p}=0.87,0.67$ and 0.99 , respectively). All but 1 participant were prescribed regular intravenous iron therapy. Excluding the 5 participants who received intravenous iron between visit 1 and visit 2 did not change the results (Additional file 1: Table S5).

\section{Change in LV ejection fraction}

There was no overall change in LVEF following dialysis (Table 1). Six participants had abnormal LVEF pre-dialysis based on age and sex standardised reference ranges [33]. In 5 of these participants, LVEF improved following dialysis and fluid removal (range 3-9.5\%). However, 11 participants with normal LVEF pre-dialysis, had abnormal LVEF post-dialysis. In one participant, a dramatic reduction in LVEF was clearly due to tachy-arrhythmia. In the remaining 10 participants, 4 had minor changes 

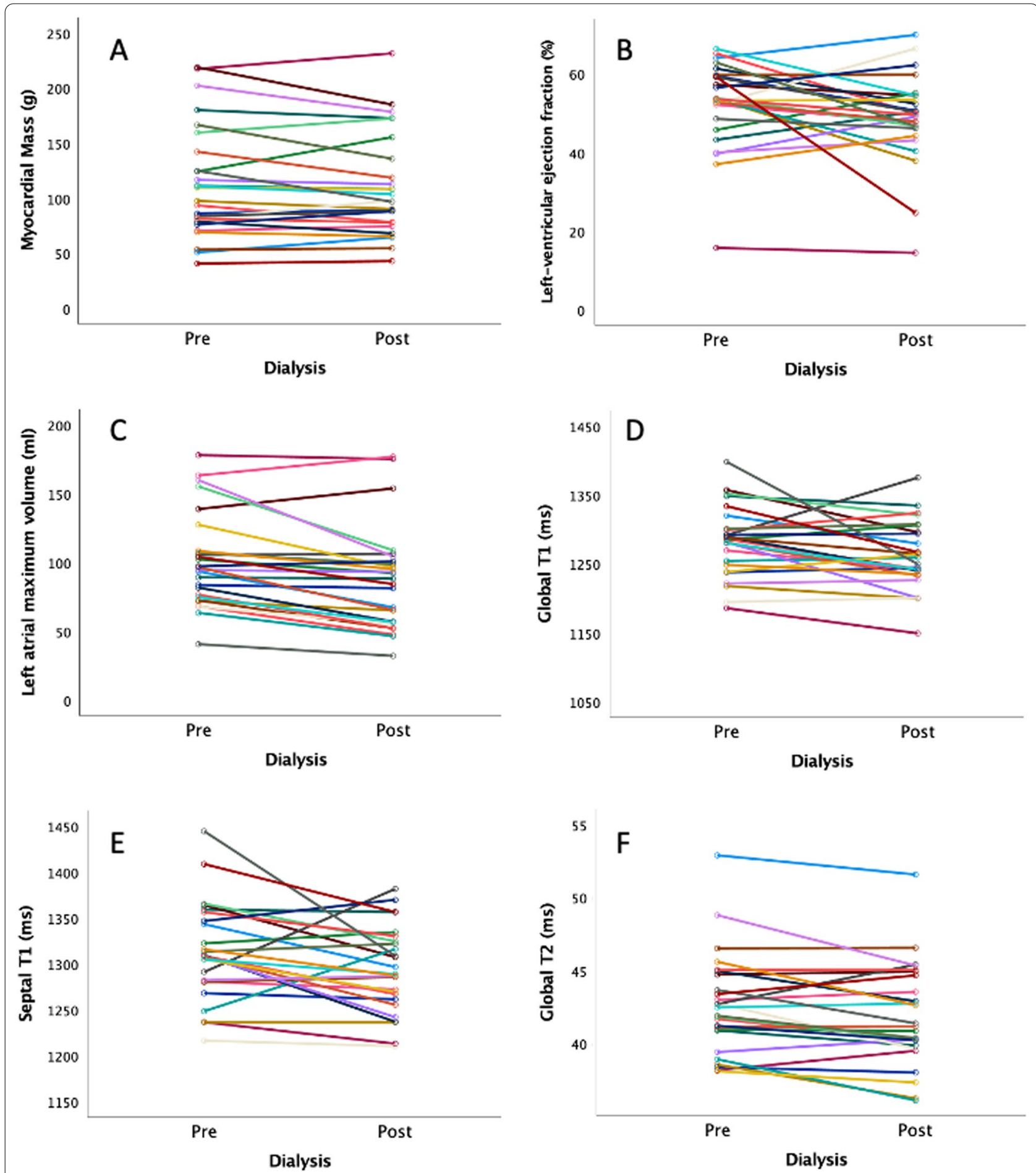

Fig. 3 Within subject changes pre- and post- dialysis for left ventricular mass (A), left ventricular ejection fraction (B), left atrial maximum volume (C), global T1 times (D), septal T1 times $(\mathbf{E})$, and global T2 times (F)

( $<5 \%$ difference) that crossed the threshold for age and sex standardised normal values, while 6 had $>5 \%$ reduction in LVEF but without obvious association between the change in LVEF and baseline hydration status (visit 1 bioimpedance hydration status ranging from -3.8 to +2.5 L). On multivariable linear regression including baseline 

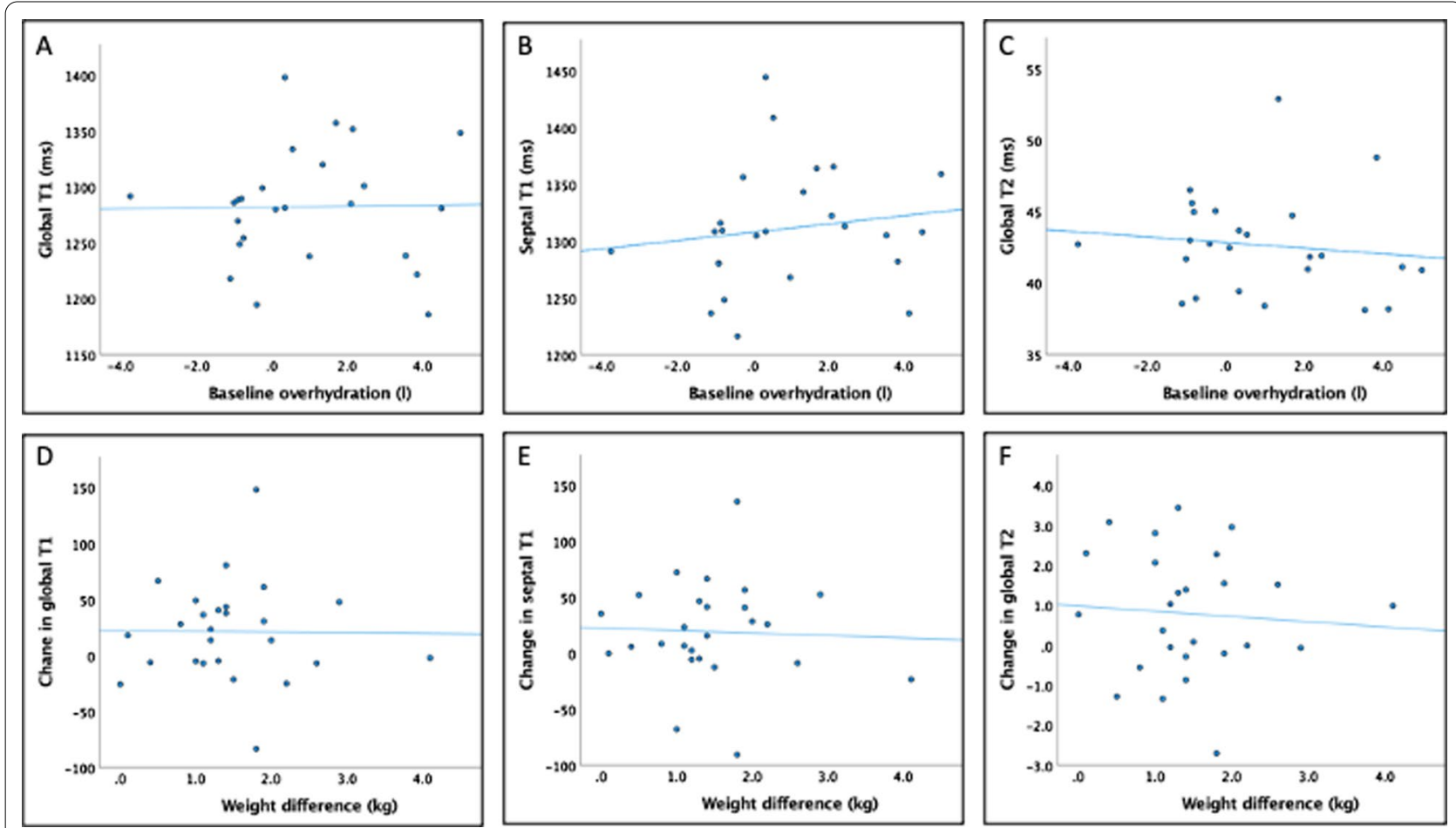

Fig. 4 Scatter plots of global T1, septal T1 and global T2 according to baseline over-hydration (A-C respectively), and the change in global T1, septal T1 and global T2 according to change in weight (D-F respectively). There was no significant association in any of the comparisons

over-hydration, baseline LVEF, ultrafiltration volume, follow-up over-hydration, over-hydration change, weight change, time from visit 1 until dialysis and time from dialysis until repeat CMR, only baseline LVEF and the time from visit 1 until dialysis significantly associated with the change in LVEF following a backwards elimination approach (baseline LVEF: Beta 0.43, $\mathrm{p}=0.02$; Time from CMR 1 until dialysis: Beta 0.38, $\mathrm{p}=0.04$; adjusted $\mathrm{r}^{2}$ for the model $=0.30$ ). At lower baseline LVEF, repeat LVEF was more likely to increase, whereas those who had a longer gap between visit 1 CMR and dialysis were more likely to have a reduction in LVEF at visit 2 (Fig. 5).

\section{Discussion}

This prospective study identified significant changes in cardiovascular parameters on 3T CMR in response to haemodialysis with fluid removal. Specifically, LV and $\mathrm{RV}$ end-diastolic volumes, stroke volumes, and atrial volumes reduced, as did global native $\mathrm{T} 1$, septal native $\mathrm{T} 1$ and global T2 times but not LV mass. There was no correlation between the change in these parameters and the change in fluid status measured by bodyweight or bioimpedance. The change in myocardial native T1 time was independent of changes in haematocrit and blood pool T1, suggesting that the observed difference is not explainable by reduced intravascular T1 time. Regardless of whether the change in native $\mathrm{T} 1$ time is due to fluid removal, or the dialysis process itself, the present results question the validity of native $\mathrm{T} 1$ mapping as a surrogate marker for myocardial fibrosis in patients on haemodialysis.

Native T1 mapping is an appealing potential biomarker for myocardial fibrosis, with proven superiority over volumes, function and late gadolinium enhancement in patients with non-ischaemic dilated cardiomyopathy [34, $35]$, and encouraging data in patients with CKD [18, 36, 37]. However, there are conflicting results from previous studies exploring the influence of fluid status on native T1 mapping in patients with CKD. Native T1 times do not alter with varying end-diastolic volumes (an indicator of changing fluid status) in patients on dialysis [21]. Similarly, a study comparing 124 dialysis patients to 137 healthy controls found that the increased myocardial native T1 times observed in patients with CKD occurred independently of changes in T2 times, suggesting that fibrosis, rather than fluid, accounts for the differences in T1. However, these patients were scanned the day after dialysis when euvolaemia is most likely [38]. Furthermore, a study of 12 patients found no change in $\mathrm{T} 1$ values on $3 \mathrm{~T}$ CMR immediately post dialysis [39]. These patients had relatively low ultrafiltration volumes (mean $1.1 \mathrm{~L}$ ) and the lack of effect could be explained by insufficient time to 

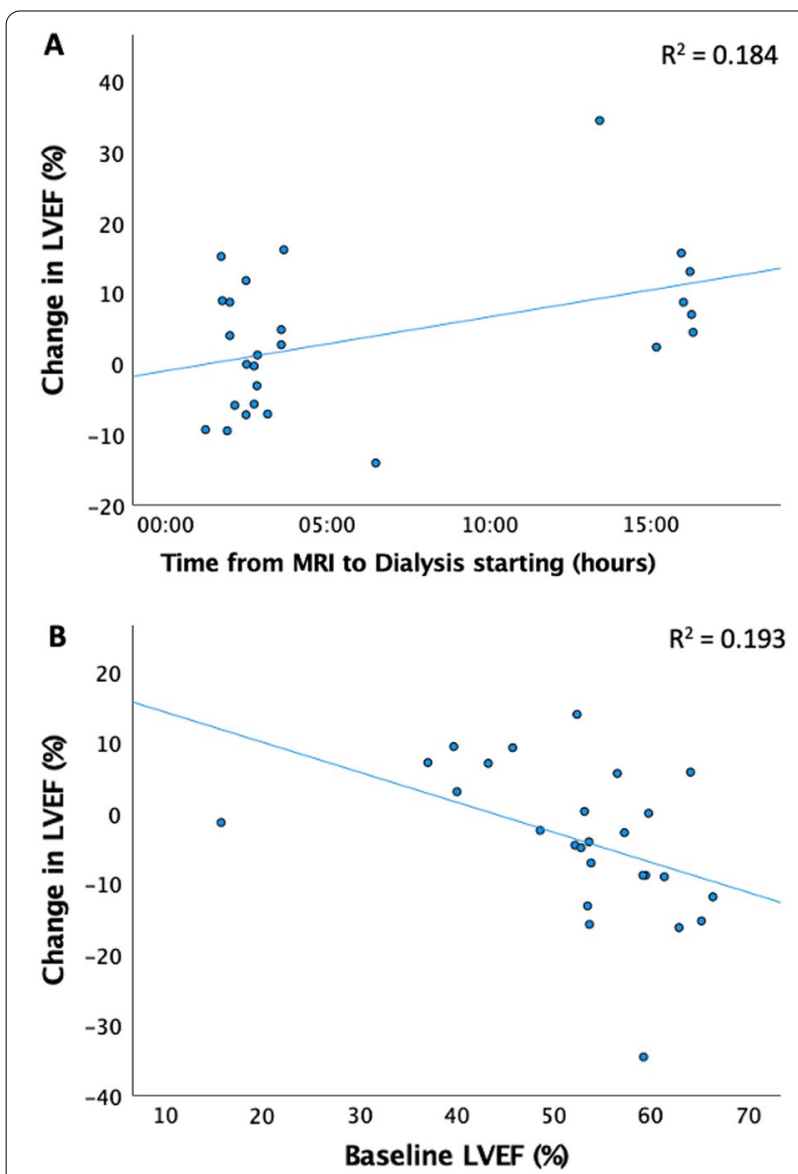

Fig. 5 Scatter plots of change in left ventricular ejection fraction (LVEF) [calculated by visit 2 post-dialysis LVEF (\%) - visit 1 pre-dialysis LVEF (\%)] by baseline LVEF $(p=0.02)(\mathbf{A})$ and time from visit 1 CMR until dialysis $(p=0.04)(B)$

allow for fluid re-equilibration. In the MIDNIGHT study [40], which found significant improvements in native T1 time on 3T CMR with extended hours nocturnal haemodialysis, there was no association between native $\mathrm{T} 1$ time and fluid status on bio-impedance body composition monitoring. However, the change in $\mathrm{T} 1$ did occur in the presence of increased ultrafiltration volumes in the treatment group, and reduced ultrafiltration volumes in the control group. In contrast, a study of 30 dialysis patients found a significant correlation $(r=0.409)$ between fluid status and native T1 time on 1.5T CMR [24]. This result could be explained by reverse causality, with patients with more myocardial fibrosis being more prone to fluid overload. Kotecha et al. found global native T1 times on 1.5T CMR reduced from $1085 \mathrm{~ms}$ pre-dialysis to $1072 \mathrm{~ms}$ post-dialysis in 25 dialysis patients undergoing a $3 \mathrm{~h}$ dialysis session with mean $2.0 \mathrm{~L}$ ultrafiltration [25]. The present study confirms this result at 3T and supports the conclusion that the abnormal native $\mathrm{T} 1$ times observed in patients with CKD can be modulated by dialysis with fluid removal and therefore is not entirely due to fibrosis.

The minimal clinically significant change in native $\mathrm{T} 1$ time is difficult to define. Previous studies comparing native $\mathrm{T} 1$ in patients on dialysis versus controls found a mean difference of $21 \mathrm{~ms}$ on $1.5 \mathrm{~T}$ [19] and $185 \mathrm{~ms}$ on $3 \mathrm{~T}$ [20]. In the present study, the mean global T1 are greater than the scanner-specific healthy reference range by a mean of $113 \mathrm{~ms}$ pre-dialysis and $91 \mathrm{~ms}$ post dialysis (Table 1). The mean change in T1 time pre- and postdialysis was $21 \mathrm{~ms}$. In the MIDNIGHT trial [40], the intervention resulted in a mean reduction in native $\mathrm{T} 1$ time of $31 \mathrm{~ms}$ (from a mean baseline value of $1270 \mathrm{~ms}$ ). As another example, in non-CKD patients undergoing aortic valve replacement, native $\mathrm{T} 1$ times reduced by an average of $45 \mathrm{~ms}$ and were associated with improved prognosis [41]. So while the difference in global T1 observed by this study, and by Kotecha et al. [25], is small, it is within the region of clinically significant difference. Similar magnitude of change has been observed in healthy volunteers and patients with coronary artery disease immediately post exercise, but in this setting native T1 times increased, rather than decreased [42]. In these patients with coronary artery disease, the magnitude of native T1 reactivity correlated with the severity of myocardial perfusion abnormality [42]. This suggests that any change in native $\mathrm{T} 1$ times following dialysis is unlikely to be due to dialysis-induced ischaemia (which would cause times to increase).

Native T1 predicts outcome in patients with heart failure [34] and acute myocardial injury [43]. It also has proven diagnostic or prognostic benefit in a range of other conditions including amyloidosis, myocarditis, aortic stenosis, iron overload, and Anderson-Fabry disease $[12,44]$. The present results question the on-going consideration of native T1 mapping as a surrogate for myocardial fibrosis in patients on haemodialysis. Studies including myocardial biopsy data will be needed to answer this definitively but would be challenging to justify ethically and difficult to complete. There is an ongoing study correlating native T1 mapping with postmortem histology in 9 participants (ClinicalTrials.gov Identifier: NCT03586518). For native T1 to proceed as a potential biomarker in CKD patients, it will require longitudinal studies with standardised timing of imaging in relation to dialysis therapy to establish if native $\mathrm{T} 1$ has a prognostic role in the CKD population, and if changes in native $\mathrm{T} 1$ times correspond with proportional changes in prognosis. If proven, the small changes in native $\mathrm{T} 1 \mathrm{fol}$ lowing dialysis may be deemed negligible.

Global T2 times reduced following dialysis with fluid removal, in keeping with previous studies [25]. The native transverse relaxation time (T2) is sensitive to proton 
(water) binding to macromolecules and proton mobility. Native T2 reflects tissue water content and mobility to a greater extent than native longitudinal relaxation time (T1). Skeletal muscle T2 times also decreased suggesting that the observed myocardial change may be due to reduced total body water content, rather than a myocardial-specific process, but there was no change in hepatic or blood pool T2. The timing of radiofrequency pulse sequence used in T2-weighted images results in increasing signal intensity with increasing water content of tissues [45], and so it is physically plausible that the change in T2 represents reduced tissue oedema. The lack of association between the change in $\mathrm{T} 2$ and the change in fluid status is against this, but it still remains the most likely explanation.

There appears to be a complex relationship with regards to parameters of ventricular function and dialysis with fluid removal. A study using intradialytic CMR has previously shown that LVEF drops acutely during dialysis with incomplete recovery evident at $1 \mathrm{~h}$ post dialysis [39]. This explains the present observation whereby the timing of dialysis and repeat CMR was a significant factor in predicting repeat LVEF (albeit with a very weak correlation), with those patients on a morning dialysis schedule (and therefore undergoing repeat CMR soon after dialysis) being more likely to have a reduction in repeat LVEF. Paradoxically, in the sub-group of patients with reduced LVEF, previous reports have suggested that dialysis with fluid removal can improve LVEF [25]. In the present study, 5 of the 6 patients with abnormal LVEF at visit 1 had an improvement on repeat LVEF measurement, presumably due to reduced afterload. With regards to clinical practice, CMR scanning should be avoided immediately post-dialysis and serial scanning should be performed at same time in relation to dialysis schedule. Given the differential response in LVEF depending on baseline LVEF, it is conceivable that the wrong dialysis prescription could perpetuate a patient's cardiac dysfunction and is a reminder of the importance of the individualised medicine in dialysis prescribing. There is increasing interest in the role of LV global longitudinal strain as a potentially superior measure of cardiac function compared to LVEF, especially in patients with CKD $[36,46]$. Encouragingly, LV global longitudinal strain did not differ pre- and post-dialysis, suggesting it may give a consistent, volume-independent assessment of cardiac function in dialysis patients.

\section{Limitations}

This study addresses important questions in relation to 3T CMR to inform timing of clinical scanning in relation to dialysis and the potential bias of fluid overload in parametric mapping. The cohort is representative of the wider dialysis population with high prevalence of comorbidity and no changes to their prescribed dialysis session. The number of clinically relevant incidental findings that were identified is striking but is in keeping with previous reports [47]. Fluid assessment was comprehensive and CMR scans were performed utilising state-of-theart hardware and software. However, there are several limitations. The sample was heterogeneous with regards to timing of scans in relation to dialysis, baseline LVEF (despite attempts to control this by excluding patients with known LV dysfunction) and baseline hydration status, with 10 participants measuring as volume deplete on bioimpedance monitoring at visit 1 . We cannot discount a Type 2 error for the lack of correlation between myocardial native T1 and fluid removal. Further, there may have been a differential time-course between changes in fluid status and native T1. The impact of $1 \mathrm{~L}$ fluid removal is likely to have differential effect on the myocardium if the starting state is volume overload, as opposed to volume depletion. Nevertheless, there was no apparent difference in change in native T1 times according to baseline hydration status. The study could have been improved by inclusion of a control group who underwent dialysis without fluid removal. Further work is warranted.

\section{Conclusion}

Acute changes in cardiac volumes and myocardial composition are detectable on 3T CMR following haemodialysis with fluid removal. Accordingly, the timing of clinical CMR scanning in relation to a patient's dialysis schedule is crucial, particularly if serial scanning is required. Small, but significant, reductions in global myocardial $\mathrm{T} 1$ and $\mathrm{T} 2$ relaxation times were observed after dialysis suggesting that the abnormal native T1 signal in patients undergoing haemodialysis is not entirely due to fibrosis. The exact mechanism for the reduction in native $\mathrm{T} 1$ is unclear. Despite the lack of association with the change in native $\mathrm{T} 1$ and the change in fluid status, alterations in tissue oedema remain the most likely explanation, albeit removal of uraemic factors or the haemodynamic effects of dialysis itself may also contribute. Future studies examining the prognostic capabilities of native T1 in CKD populations are still warranted but will require careful standardisation of imaging schedules and awareness of the potential confounding effect of fluid status and the dialysis process.

\section{Abbreviations}

CKD: Chronic kidney disease; CMR: Cardiovascular magnetic resonance; CVD: Cardiovascular disease; ECG: Electrocardiogram; LA: Left atrium/left atrial; LV: Left ventricle/left ventricular; LVEF: Left ventricular ejection fraction; RA: Right atrium/right atrial; RV: Right ventricle/right ventricular. 


\section{Supplementary Information}

The online version contains supplementary material available at https://doi. org/10.1186/s12968-021-00822-4.

Additional file 1. Supplementary material: $\mathbf{S 1}$. Baseline characteristics of participants. S2. Summary of clinically significant incidental findings. S3. Intra- and inter-observer reproducibility for cardiovascular MRI parameters. S4. Determinants of blood pool native T1. S5. Analyses exploring the potential influence of intravenous iron therapy on native T1.

\section{Acknowledgements}

We wish to thank all participants who kindly volunteered their time for this study. We also wish to thank Dr Andrew Morrow for providing the scannerspecific reference ranges for myocardial native $\mathrm{T} 1$ and $\mathrm{T} 2$ in healthy individuals.

\section{Authors' contributions}

All authors have reviewed and contributed to this manuscript. PBM, GR, AJR, ER and KM conceived the idea and analysis plan for this study. AJR recruited participants, performed study visits, analysed the CMRs, analysed the data and wrote the manuscript. KM, GR, TT and CB advised on CMR analysis and critically reviewed the manuscript. KM also analysed a sample of CMRs for inter-observer reproducibility. EE analysed data regarding the determinants of blood pool native T1. LD led image acquisition. KAG and JSL assisted with data collection, analysis and critically reviewed the manuscript. PBM, ER, RKP and AR advised on analysis plan and critically reviewed this manuscript. All authors read and approved the final manuscript.

\section{Funding}

Dr Alastair Rankin is personally funded by a Clinical Academic Training Fellowship from The Chief Scientist Office (Scotland) (CAF/18/02). Dr Elaine Rutherford is supported by a Postdoctoral Clinical Lectureship from The Chief Scientist Office (Scotland) (PCL/18/03). Dr Jennifer Lees is supported by a Postdoctoral Clinical Lectureship from The Chief Scientist Office (Scotland) (PCL/20/10). Thomas Treibel is currently supported by a BHF Intermediate fellowship (FS/19/35/34374). Colin Berry is funded by a British Heart Foundation Centre of Excellence Award (RE/18/6/34217).

\section{Availability of data and materials}

Available via the corresponding author upon reasonable request.

\section{Declarations}

\section{Ethics approval and consent to participate}

All participants gave written consent prior to participation. Favourable ethical opinion was granted by the West of Scotland Research Ethics Committee 1 (Ref: 18/WS/0138, 13th August 2018).

\section{Consent for publication}

All participants gave written consent for their data and/or images to be published as part of a scientific report.

\section{Competing interests}

The authors declare no competing interests relevant to the present study. Outside the present study: Patrick Mark reports speaker honoraria from ViforFresenius, Astra Zeneca, Janssen, Napp, Novartis and Bristol Myers-Squibb, research grants from Boehringer Ingelheim and non-financial support from Pharmacosmos. Jennifer Lees reports speaker honoraria from Vifor-Fresenius, Astra Zeneca, Bristol Myers-Squibb and Pfizer. Colin Berry is employed by the University of Glasgow which holds consultancy and research agreements with Abbott Vascular, AstraZeneca, Boehringer Ingelheim, Causeway Therapeutics, Coroventis, Genentech, GSK, HeartFlow, Menarini, and Siemens Healthcare.

\section{Author details}

${ }^{1}$ Institute of Cardiovascular and Medical Sciences, University of Glasgow, 126 University Place, Glasgow G12 8TA, UK. ${ }^{2}$ Renal and Transplant Unit, NHS Greater Glasgow and Clyde, Glasgow, UK. ${ }^{3}$ Clinical Research Imaging, NHS Greater Glasgow and Clyde, Glasgow, UK. ${ }^{4}$ Institute for Cardiovascular Sciences and Barts Heart Centre, University College London, London, UK. ${ }^{5}$ Department of Radiology, NHS Greater Glasgow and Clyde, Glasgow, UK.

Received: 23 September 2021 Accepted: 18 October 2021

Published online: 11 November 2021

\section{References}

1. Baigent C, Burbury K, Wheeler D. Premature cardiovascular disease in chronic renal failure. Lancet. 2000;356:147-52.

2. Go AS, Chertow GM, Fan D, McCulloch CE, Hsu C. Chronic kidney disease and the risks of death, cardiovascular events, and hospitalization. N Engl J Med. 2004;351:1296-305. https://doi.org/10.1056/NEJMoa041031.

3. Matsushita K, van der Velde M, Astor BC, Woodward M, Levey AS, de Jong $P E$, et al. Association of estimated glomerular filtration rate and albuminuria with all-cause and cardiovascular mortality in general population cohorts: a collaborative meta-analysis. Lancet. 2010;375:2073-81.

4. Foley RN. Chronic kidney disease and the risk for cardiovascular disease, renal replacement, and death in the United States medicare population, 1998 to 1999. J Am Soc Nephrol. 2005;16:489-95. https://doi.org/10. 1681/ASN.2004030203.

5. Methven S, Steenkamp R, Fraser S. UK renal registry 19th annual report: chapter 5 survival and causes of death in UK adult patients on renal replacement therapy in 2015: national and centre-specific analyses. Nephron. 2017;137(Suppl 1):117-50.

6. Saravanan P, Davidson NC. Risk assessment for sudden cardiac death in dialysis patients. Circ Arrhythm Electrophysiol. 2010;3:553-9.

7. USRDS. USRDS annual data report: epidemiology of kidney disease in the United States. Am J Kidney Dis. 2016;2017(69):S659-88.

8. Herzog CA. Cardiac arrest in dialysis patients: approaches to alter an abysmal outcome. Kidney Int Suppl. 2003;63:S197-200.

9. Edwards NC, Moody WE, Chue CD, Ferro CJ, Townend JN, Steeds RP. Defining the natural history of uremic cardiomyopathy in chronic kidney disease: the role of cardiovascular magnetic resonance. JACC CardiovasC Imaging. 2014;7:703-14.

10. Aoki J, Ikari Y, Nakajima H, Mori M, Sugimoto T, Hatori M, et al. Clinical and pathologic characteristics of dilated cardiomyopathy in hemodialysis patients. Kidney Int. 2005;67:333-40.

11. Mall G, Huther W, Schneider J, Lundin P, Ritz E. Diffuse intermyocardiocytic fibrosis in uraemic patients. Nephrol Dial Transplant. 1990;5:39-44

12. Graham-Brown MPM, Patel AS, Stensel DJ, March DS, Marsh A-M, McAdam J, et al. Imaging of myocardial fibrosis in patients with endstage renal disease: current limitations and future possibilities. Biomed Res Int. 2017;2017:1-14.

13. Mark PB, Johnston N, Groenning BA, Foster JE, Blyth KG, Martin TN, et al. Redefinition of uremic cardiomyopathy by contrast-enhanced cardiac magnetic resonance imaging. Kidney Int. 2006;69:1839-45.

14. Schietinger BJ, Brammer GM, Wang H, Christopher JM, Kwon KW, Mangrum AJ, et al. Patterns of late gadolinium enhancement in chronic hemodialysis patients. JACC Cardiovasc Imaging. 2008;1:450-6.

15. Collidge TA, Thomson PC, Mark PB, Traynor JP, Jardine AG, Morris STW, et al. Gadolinium-enhanced MR imaging and nephrogenic systemic fibrosis: retrospective study of a renal replacement therapy cohort. Radiology [Internet]. 2007;245:168-75. https://doi.org/10.1148/radiol.24510 70353.

16. Bruce R, Wentland AL, Haemel AK, Garrett RW, Sadowski DR, Djamali A, et al. Incidence of nephrogenic systemic fibrosis using gadobenate dimeglumine in 1423 patients with renal insufficiency compared with gadodiamide. Invest Radiol. 2016;51:701-5.

17. Badve SV, Palmer SC, Strippoli GFM, Roberts MA, Teixeira-Pinto A, Boudville N, et al. The validity of left ventricular mass as a surrogate end point for all-cause and cardiovascular mortality outcomes in people with CKD: a systematic review and meta-analysis. Am J Kidney Dis [Internet]. 2016;68:554-63. https://doi.org/10.1053/j.ajkd.2016.03.418.

18. Taylor AJ, Salerno M, Dharmakumar R, Jerosch-Herold M. T1 Mapping basic techniques and clinical applications [Internet]. JACC CardiovasC. Imaging. 2016

19. Rutherford E, Talle MA, Mangion K, Bell E, Rauhalammi SM, Roditi G, et al. Defining myocardial tissue abnormalities in end-stage renal failure with 
cardiac magnetic resonance imaging using native T1 mapping. Kidney Int. 2016;90:1-8.

20. Graham-Brown MPM, March DS, Churchward DR, Stensel DJ, Singh A, Arnold R, et al. Novel cardiac nuclear magnetic resonance method for noninvasive assessment of myocardial fibrosis in hemodialysis patients. Kidney Int. 2016;90:835-44.

21. Graham-Brown MPM, Rutherford E, Levelt E, March DS, Churchward DR, Stensel DJ, et al. Native T1 mapping: inter-study, inter-observer and intercenter reproducibility in hemodialysis patients. J Cardiovasc Magn Reson. 2017;19:21.

22. Bull S, White SK, Piechnik SK, Flett AS, Ferreira VM, Loudon M, et al. Human non-contrast T1 values and correlation with histology in diffuse fibrosis. Heart. 2013;99:932-7. https://doi.org/10.1136/heartjnl-2012-303052.

23. de Meester de RC, Bouzin C, Lazam S, Boulif J, Amzulescu M, Melchior J, et al. Histological Validation of measurement of diffuse interstitial myocardial fibrosis by myocardial extravascular volume fraction from Modified Look-Locker imaging (MOLLI) T1 mapping at 3 T. J Cardiovasc Magn Reson. 2015;17:48.

24. Antlanger M, Aschauer S, Kammerlander AA, Duca F, Säemann MD, Bonderman $\mathrm{D}$, et al. Impact of systemic volume status on cardiac magnetic resonance T1 mapping.

25. Kotecha T, Martinez-Naharro A, Yoowannakul S, Lambe T, Rezk T, Knight DS, et al. Acute changes in cardiac structural and tissue characterisation parameters following haemodialysis measured using cardiovascular magnetic resonance. Sci Rep. 2019;9:1-8. https://doi.org/10.1038/ s41598-018-37845-4.

26. Dill T. Contraindications to magnetic resonance imaging [Internet]. Heart 2008.

27. Board of the faculty of Clinical Radiology. Standards for intravascular contrast agent administration to adult patients Second edition. R Coll Clin Radiol. 2011; 3-15.

28. Schulz-Menger J, Bluemke DA, Bremerich J, Flamm SD, Fogel MA, Friedrich MG, et al. Standardized image interpretation and post processing in cardiovascular magnetic resonance: Society for Cardiovascular Magnetic Resonance (SCMR) Board of Trustees Task Force on Standardized Post Processing. J Cardiovasc Magn Reson 2013;15.

29. Cerqueira MD, Weissman NJ, Dilsizian V, Jacobs AK, Kaul S, Laskey WK, et al. Standardized myocardial sementation and nomenclature for tomographic imaging of the heart: a Statement for Healthcare Professionals from the Cardiac Imaging Committee of the Council on Clinical Cardiology of the American Heart Association [Internet]. Circulation. 2002. https://doi.org/10.1161/hc0402.102975.

30. Captur G, Gatehouse P, Keenan KE, Heslinga FG, Bruehl R, Prothmann M, et al. A medical device-grade T1 and ECV phantom for global T1 mapping quality assurance - the T1 Mapping and ECV Standardization in cardiovascular magnetic resonance (T1MES) program. J Cardiovasc Magn Reson. 2016;18:58.

31. Charlson ME, Pompei P, Ales KL, Mackenzie CR. A new method of classifying prognostic comorbidity in longitudinal studies: development and validation. J Chronic Dis. 1987;40:373-83.

32. Hemmelgarn BR, Manns BJ, Quan H, Ghali WA. Adapting the Charlson comorbidity index for use in patients with ESRD. Am J Kidney Dis. 2003:42:125-32.

33. Petersen SE, Aung N, Sanghvi MM, Zemrak F, Fung K, Paiva JM, et al. Reference ranges for cardiac structure and function using cardiovascular magnetic resonance (CMR) in Caucasians from the UK Biobank population cohort. J Cardiovasc Magn Reson 2017;19.

34. Puntmann VO, Carr-White G, Jabbour A, Yu CY, Gebker R, Kelle S, et al. T1-mapping and outcome in nonischemic cardiomyopathy all-cause mortality and heart failure. JACC Cardiovasc Imaging. 2016;9:40-50.
35. Puntmann VO, Valbuena S, Hinojar R, Petersen SE, Greenwood JP, Kramer CM, et al. Society for Cardiovascular Magnetic Resonance (SCMR) expert consensus for CMR imaging endpoints in clinical research: part i-analytical validation and clinical qualification [Internet]. J Cardiovasc Magn Reson 2018. http://creativecommons.org/publicdomain/zero/1.0/.

36. Mangion K, McDowell K, Mark PB, Rutherford E. Characterizing cardiac involvement in chronic kidney disease using CMR - a systematic review [Internet]. Curr Cardiovasc Imaging Rep. 2018. https://doi.org/10.1007/ s12410-018-9441-9.

37. Haaf P, Garg P, Messroghli DR, Broadbent DA, Greenwood JP, Plein S. Cardiac T1 mapping and extracellular volume (ECV) in clinical practice: a comprehensive review. J Cardiovasc Magn Reson. 2016;18:89.

38. Graham-Brown MPM, Gulsin GS, Poli F, Parke K, Burton JO, McCann GP. Differences in native $\mathrm{T} 1$ and native T2 mapping between patients on hemodialysis and control subjects. Eur J Radiol. 2021;140:109748.

39. Buchanan C, Mohammed A, Cox E, Köhler K, Canaud B, Taal MW, et al. Intradialytic cardiac magnetic resonance imaging to assess cardiovascular responses in a short-term trial of hemodiafiltration and hemodialysis. J Am Soc Nephrol. 2017;28:1269-77. https://doi.org/10.1681/ASN.20160 60686.

40. Graham-Brown MPM, Churchward DR, Hull KL, Preston R, Pickering WP, Eborall HC, et al. Cardiac remodelling in patients undergoing in-centre nocturnal haemodialysis: results from the MIDNIGHT Study, a non-randomized controlled trial. Blood Purif. 2017;44:301-10.

41. Hwang IC, Kim HK, Park JB, Park EA, Lee W, Lee SP, et al. Aortic valve replacement-induced changes in native $\mathrm{T} 1$ are related to prognosis in severe aortic stenosis: $\mathrm{T1}$ mapping cardiac magnetic resonance imaging study. Eur Heart J Cardiovasc Imaging. 2020;21:653-63.

42. Nakamori S, Fahmy A, Jang J, El-Rewaidy H, Neisius U, Berg S, et al. Changes in myocardial native T 1 and $\mathrm{T} 2$ after exercise stress a noncontrast CMR pilot study. JACC Cardiovasc Imaging. 2019. https://doi.org/10. 1016/j.jcmg.2019.05.019.

43. Liu D, Borlotti A, Viliani D, Jerosch-Herold M, Alkhalil M, De Maria GL, et al. CMR native T1 mapping allows differentiation of reversible versus irreversible myocardial damage in ST-segment-elevation myocardial infarction: an OxAMI Study (Oxford Acute Myocardial Infarction). Circ Cardiovasc Imaging. 2017. https://doi.org/10.1161/CIRCIMAGING.116. 005986/-/DC1.

44. Messroghli DR, Moon JC, Ferreira VM, Grosse-Wortmann L, He T, Kellman $P$, et al. Clinical recommendations for cardiovascular magnetic resonance mapping of T1, T2, T2 and extracellular volume: a consensus statement by the Society for Cardiovascular Magnetic Resonance (SCMR) endorsed by the European Association for Cardiovascular Imaging. J Cardiovasc Magn Reson. 2017;19:1-24.

45. Bloem JL, Reijnierse M, Huizinga TWJ, Van Der Helm-Van Mil AHM. MR signal intensity: staying on the bright side in MR image interpretation. RMD Open. 2018;4:728.

46. Rankin AJ, Zhu L, Mangion K, Rutherford E, Gillis KA, Lees JS, et al. Global longitudinal strain by feature-tracking cardiovascular magnetic resonance imaging predicts mortality in patients with end-stage kidney disease. Clin Kidney J. 2021. https://doi.org/10.1093/ckj/sfab020/6126373.

47. Rutherford E, Weir-McCall JR, Patel RK, Houston JG, Roditi G, Struthers $A D$, et al. Research cardiac magnetic resonance imaging in end stage renal disease-incidence, significance and implications of unexpected incidental findings. Eur Radiol Springer Verlag. 2017;27:315-24.

\section{Publisher's Note}

Springer Nature remains neutral with regard to jurisdictional claims in published maps and institutional affiliations. 